\title{
Professional Issues and Trends in Nursing in Japan
}

\section{Akiko Kondo*}

School of Nursing, Graduate School of Nursing, Tokyo Women's Medical University, Tokyo, Japan

Current important topics in nursing care and medical society in Japan are cancer care, elder care, and effective use health care resources in an aging society as well as in the general population in the same fashion as other developed countries. This special issue includes 6 articles related to these topics, from elders to children, focusing on specific care, administration and the nurses' work environment.

One of the highest causes of death currently in Japan is cancer, especially for those from 40 to 89 years of age [1]. Among cancer, lung cancer has the highest fatality, and the mortality of lung cancer is increasing [2]. This special issue introduces Horii et al. [3] article about cancer nursing. This study describes life adjustment in patients with a diagnosis of lung cancer whose performance status is decreased.

The percentage of people who are 65 years and older in Japan increased to more than $24 \%$ in 2012 , because the first group of "baby boomers" became 65 years old [4]. Incidence of hip fracture increases as the population ages. Hip fracture is one of the main causes of being bedridden, which leads to social and economic problems. It is important to prevent being bed-ridden and to make elders healthy. Kondo et al. [5] details a study that shows nursing care soon after hip fracture surgery can improve patients' short-term and long-term outcomes such as ambulatory ability after discharge.

It is necessary to use limited health resources effectively and to provide safe care with high quality in such an aging society. Appropriate deployment of nursing staff is important to use personnel effectively and to secure quality of nursing care. However, it has been a challenge for nurse managers to do so. Mashimo et al. [6] clarifies topics of appropriate nursing staffing and finds points of improvement for Japan's patient classification system by interviewing nurse administrators.

Arranging a better work environment is indispensable to maintain satisfactory numbers of nurses in hospitals so that safe care with high quality is provided to patients. Tei-Tominaga et al. [7] examined the effect of characteristics of the work environment of Magnet hospitals on nurses' job satisfaction.

Many developed countries have been introducing nurse practitioners. However, this practice is still controversial in Japan. The current law allows diagnosis and prescription only for medical doctors and dentists. Certified Nurse Specialists and Certified Nurses were institutionalized in 1996. Certified Nurse Specialists and Certified Nurses are allowed to practice as established in the Act on Public Health Nurses, Midwives and Nurses, which initially acted in 1948 and whose final revision was in 2009. One article describes the education of such advanced practice registered nurses and the related issues and obstacles of introducing nurse practitioner system in Japan [8].

Japanese adolescents giving birth to their babies are increasing although the rate is low. They chose to give birth more often instead of abortion than in past years. It is important to encourage having children in order to relieve the aging population. However, it is sometimes hard for teenagers to be good parents and to interact with their children. Komoto et al. [9] examined the adolescent mother-infant interaction, maternal self-esteem, and parenting stress, which affects child development, in order to make recommendations for helping Japanese adolescent mothers in parenting.

I really appreciate the editors of the Journal of Nursing and Care for providing the opportunity to participate in this special issue. Please enjoy!

\section{References}

1. Ministry of Health Labour and Welfare (2009) Table 8: Order of cause of death http://www.mhlw.go.jp/toukei/saikin/hw/jinkou/suii09/deth8.html [Accessed October 28, 2012 (in Japanese)].

2. Ministry of Health Labour and Welfare (2009) Mortality. http://www.mhlw.go.jp/ toukei/saikin/hw/jinkou/geppo/nengai09/kekka3.html [Accessed October 28, 2012 (in Japanese)].

3. Horii N, Kondo A, Ejiri H, Maekawa A (2012) Structure of Life Adjustments by Lung Cancer Patients with Decreased Performance Status. J Nurs Care S5001: doi. 10.4172/2167-1168.S5-001.

4. MSN Sankei News (2012) "Baby boomer" became elders, nubmer of 65 years and older reached more than $30,000,000$ from the assumption of Ministry of Internal Affairs and Communications http://sankei.jp.msn.com/life/ news/120916/trd12091617180004-n1.htm [Accessed October 28, 2012].

5. Kondo A, Sada K, Yamaguchi C, Fujimoto E (2012) Nursing Care after Hip Fracture Surgery Predicts Patient Ambulatory Ability at 3 Months after Surgery. J Nurs Care S5-002. doi: 10.4172/2167-1168.S5-002.

6. Mashimo A, Akiyama T, Fukui T, Ueno M,Tanaka A (2013) Survey of the Nurse Staffing System Including the Patient Classification System and Issues in Nurse Resource Management in Japan. Journal of Nursing and Care S5-005. doi: 10.4172/2167-1168.S5-005.

7. Tei-Tominaga M, Tsuchiya M, Sato F (2012) Characteristics of the Work Environment of Magnet Hospitals and Job Satisfaction among Nurses in Japan: A Cross-Sectional Study Using Multi- Level Analysis. J Nurs Care S5-003. doi: 10.4172/2167-1168.S5-003.

8. Kondo A (2013) Advanced Practice Nurses in Japan: Education and Related Issues. Journal of Nursing and Care S5-004. doi: 10.4172/2167-1168.S5-004.

9. Komoto K, Hirose T, Okamitsu M (2013) Nursing Intervention in Infant Mental Health: Enhancing Mother-Infant Interaction and Self-Esteem of Adolescent Mothers. J Nurs Care S5-006. doi: 10.4172/2167-1168.S5-006.
*Corresponding author: Akiko Kondo, School of Nursing, Graduate School of Nursing, Tokyo Women's Medical University, Tokyo, Japan, E-mail: kondo.akiko@twmu.ac.jp

Received May 29, 2013; Accepted June 05, 2013; Published June 07, 2013

Citation: Kondo A (2013) Professional Issues and Trends in Nursing in Japan. J Nurs Care S5:e001. doi:10.4172/2167-1168.S5-e001

Copyright: (c) 2013 Kondo A. This is an open-access article distributed under the terms of the Creative Commons Attribution License, which permits unrestricted use, distribution, and reproduction in any medium, provided the original author and source are credited. 